\title{
Weight changes after total knee arthroplasty in Chinese patients: a matched cohort study regarding predictors and outcomes
}

\author{
Pengfei Zan ${ }^{1,2}$, Jie J. Yao ${ }^{3}$, Kaiyuan Liu², Dong Yang ${ }^{2}$, Weixu Li ${ }^{1^{*}}$ and Guodong $\mathrm{Li}^{2^{*}}$
}

\begin{abstract}
Background: The purpose of this study was to compare 2-year BMI changes between patients undergoing simultaneous bilateral total knee arthroplasty (TKA), staged bilateral TKA, and unilateral TKA. We also sought to determine the predictors of weight change and whether clinically meaningful weight changes affected outcomes.

Patients and methods: This retrospective, single-institution study included 202 Chinese patients who received simultaneously bilateral TKA, staged bilateral TKA, or unilateral TKA from 2008 to 2015. There were 49 simultaneous bilateral TKAs, 52 staged bilateral TKAs, and a matched 101 unilateral TKAs.

Results: 66.8\% (135/202) of patients lost weight after TKA surgery. However, 20.7\% (42/202) of patients experienced clinically meaningful weight loss (a BMI decrease of more than 5\%). Paired $t$ test showed that 2-year BMI was significantly lower than preoperative BMI $(p<0.001)$. Weight loss was significantly different between the surgical strategy $(p<0.001)$. Preoperative BMI and age were predictive of clinically significant weight loss or gain $(p<0.05)$. Multiple linear regression showed that post-operative weight loss was associated with better Western Ontario and McMaster Universities Osteoarthritis Index and SF-36 scores $(p<0.001)$.

Conclusion: Patients after TKA experience weight loss. Age and preoperative BMI predict clinically meaningful weight change. Simultaneous bilateral TKA is associated with higher likelihood of weight loss. Clinically meaningful weight loss experiences better patient-reported outcomes.
\end{abstract}

Keywords: Total knee arthroplasty, Body mass index, Weight change, WOMAC score, SF-36 score

\section{Background}

Total knee arthroplasty (TKA) is a successful, safe, and cost-effective procedure for reducing pain and restoring function in patients with end-stage knee osteoarthritis [1-4]. Although TKA is effective in obese patients, obesity is associated with prolonged hospital stay $[5,6]$, increased costs [6,7], increased blood loss [8], and an increased risk of complications, including surgical site

\footnotetext{
*Correspondence: zrlwx@zju.edu.cn; litrue2004@163.com

'Department of Orthopedic Surgery, Second Affiliated Hospital of Zhejiang University, School of Medicine, 88 Jiefang Road, Hangzhou 310009, People's Republic of China

${ }^{2}$ Department of Orthopedic Surgery, The Tenth People's Hospital Affiliated to Tongji University, 301 Yanchang Rd, Shanghai 200072, People's Republic of China

Full list of author information is available at the end of the article
}

infection [9-14], deep infection [15], and venous thromboembolism $[16,17]$. It has also been estimated that more than half of TKA patients have a body mass index (BMI) $>30$ in Western countries [18]. Recently, a workgroup of the American Association of Hip and Knee Surgeons Evidence-Based Committee [19] has published a review regarding the effects of obesity on total joint arthroplasty, documenting the adverse effects of obesity in total joint arthroplasty patients and advising that patients with a high BMI receive additional counseling regarding possible increased risk of weight-related complications.

Although many studies have described the effects of obesity on TKA, BMI changes following primary TKA are more controversial $[2,20-26]$. One recent systemic

(C) The Author(s). 2019 Open Access This article is distributed under the terms of the Creative Commons Attribution 4.0 International License (http://creativecommons.org/licenses/by/4.0/), which permits unrestricted use, distribution, and 
review [27] found that a limited number of studies properly addressed the weight changes following TKA and that additional investigation is necessary. To the best of our knowledge, there is no study investigating the BMI changes among patients when stratified by bilateral simultaneous or staged TKA versus unilateral TKA. Furthermore, the vast majority of studies have focused on Western cohorts. Due to differences in racial composition, culture, dietary habits, and esthetic standards between countries, it is essential to document BMI changes following TKA in a Chinese cohort.

Therefore, we were interested in exploring if Chinese patients lose weight after TKA; the different patterns of BMI changes among simultaneous bilateral, staged bilateral, and unilateral TKA patients; and how postoperative BMI changes affect patient-reported 2-year Western Ontario and McMaster Universities Osteoarthritis Index (WOMAC) and SF-36 scores. We hypothesized that TKA surgery will result in 2-year weight loss after TKA surgery; that simultaneous bilateral, staged bilateral, and unilateral TKA patients will experience similar weight loss; and that TKA patients with a clinically meaningful weight loss would have better patient-reported outcome scores than other patients.

\section{Patients and methods}

This retrospective study was designed using prospectively collected data of 202 patients (303 TKAs) who had their TKAs from January 1, 2008, to April 1, 2015. There were 49 simultaneous bilateral TKAs and 52 staged bilateral TKAs at our institution. These were matched in a 1:1 ratio to unilateral TKAs by age ( \pm 5 years), sex, year of operation, and preoperative BMI $\left( \pm 1 \mathrm{~kg} / \mathrm{m}^{2}\right)$. All patients had 2 years or greater follow-up. Patients were excluded if they had a diagnosis of a systemic inflammatory disease. For staged bilateral TKA patients, patients were excluded if the interval between surgeries was more than 1 year. Finally, because we wanted to determine BMI changes and the effect on the clinical outcomes only after the successful primary TKA surgeries, we excluded those TKA patients that required any revision TKA. The study was approved by the Human Research Ethics Committee of our hospital, and all methods were performed in accordance with the relevant guidelines and regulations.

Clinical and demographic data was collected in detail before surgery. Patients' height and weight at admission, 1 year after surgery, and 2 years after surgery were collected. For patients receiving staged bilateral TKA, we used the admission data from of first TKA for analysis. BMI was calculated using the formula $\mathrm{BMI}=$ (weight in $\mathrm{kg}$ ) $/$ (height in meters $)^{2}$. We also calculated Western Ontario and McMaster
Universities Osteoarthritis Index (WOMAC) [28] scores (including WOMAC pain score, WOMAC stiffness score, WOMAC function score) as this score is the most commonly used standardized questionnaire to evaluate the condition of patients' joints. We also calculated 36-item short form health survey (SF-36) [29] scores. The SF-36 scores were also separated by the Physical Component Summary (PCS) and Mental Component Summary (MCS) scores. The SF-36 is an easily administered quality-of-life measures which can evaluate the patients' overall physical and mental functional status. Both the WOMAC and SF-36 were collected in detail at admission and two postoperative years.

Based on the World Health Organization's established BMI classification [30], patients were divided into 6 categories: underweight $\left(<18.50 \mathrm{~kg} / \mathrm{m}^{2}\right)$, normal weight (18.50 to $\left.24.99 \mathrm{~kg} / \mathrm{m}^{2}\right)$, overweight (25.00 to $29.99 \mathrm{~kg} /$ $\left.\mathrm{m}^{2}\right)$, obese class I $\left(30.00\right.$ to $\left.34.99 \mathrm{~kg} / \mathrm{m}^{2}\right)$, obese class II $\left(35.00\right.$ to $\left.39.99 \mathrm{~kg} / \mathrm{m}^{2}\right)$, and obese class III $\left(\geq 40 \mathrm{~kg} / \mathrm{m}^{2}\right)$. Within our cohort, 70 patients were normal weight, 110 patients were overweight, 20 patients were obese class I, and 2 patients were obese class II (Table 1). No patients were underweight or obese class III. Weight change was calculated as the difference of BMI at 2 years follow-up measurements compared to preoperative BMI. As defined by the US Food and Drug Administration (FDA) [31], a change in BMI of $5 \%$ or more can be considered clinically meaningful. Based on this criteria, our patients were also stratified into 3 groups: weight loss $(a>5 \%$ decrease in BMI), maintaining a stable BMI ( $a \leq 5 \%$ change in BMI), and weight gain ( $a>5 \%$ increase in BMI). We then analyzed the weight change and its effect on outcome measures within each subgroup.

\section{Statistical methods}

Descriptive statistics were performed for patients' baseline demographic characteristics. All data analysis was performed by using SPSS statistics software (SPSS Inc., Chicago, USA). Categorical variables were presented as absolute numbers and relative frequencies. Paired Student $t$ tests were used to compare 2-year BMI versus preoperative BMI. This was done both for all TKA patients and for TKA patients stratified by surgical strategy. Kruskal-Wallis $\mathrm{H}$ test and Tukey's HSD were used to evaluate differences in BMI change between TKA patient surgical strategy groups. Binomial logistic regression was used to identify the potential predictors for weight gain and loss. Multiple linear regression analysis was used to examine the effect of 2-year weight change after surgery on patient-reported outcomes, controlling for the preoperative age, sex, BMI, and Charlson-Deyo comorbidity index. A $p$ value less than 0.05 was considered as significant difference. 
Table 1 Baseline demographics and clinical characteristics of the patient population

\begin{tabular}{|c|c|c|c|c|}
\hline Parameters & Simultaneous bilateral TKA $(n=49)$ & Staged bilateral TKA $(n=52)$ & Unilateral TKA $(n=101)$ & All TKA patients $(n=202)$ \\
\hline Age (years) & $68.9 \pm 7.9$ & $68.9 \pm 7.1$ & $69.8 \pm 6.7$ & $69.4 \pm 7.1$ \\
\hline Male/female & $17 / 32$ & $20 / 32$ & $37 / 64$ & $74 / 1128$ \\
\hline Height (m) & $1.65 \pm 0.07$ & $1.64 \pm 0.08$ & $1.65 \pm 0.07$ & $1.65 \pm 0.07$ \\
\hline Weight (kg) & $70.98 \pm 8.55$ & $70.49 \pm 7.25$ & $72.31 \pm 8.95$ & $71.52 \pm 8.44$ \\
\hline $\mathrm{BMI}\left(\mathrm{kg} / \mathrm{m}^{2}\right)$ & $26.15 \pm 2.74$ & $26.45 \pm 3.43$ & $26.49 \pm 3.14$ & $26.40 \pm 3.12$ \\
\hline \multicolumn{5}{|l|}{ BMl category } \\
\hline Normal weight (18.50-24.99) & 17 (34.7\%) & $21(40.4 \%)$ & $32(31.7 \%)$ & $70(34.7 \%)$ \\
\hline Overweight (25.00-29.99) & $29(59.2 \%)$ & $23(44.2 \%)$ & $58(57.4 \%)$ & $110(54.5 \%)$ \\
\hline Obese I (30.00-34.99) & $3(6.1 \%)$ & $8(15.4 \%)$ & $9(8.9 \%)$ & $20(9.9 \%)$ \\
\hline Obese class II (35.00-35.99) & 0 & 0 & $2(2.0 \%)$ & $2(1.0 \%)$ \\
\hline \multicolumn{5}{|l|}{ Charlson-Deyo comorbidity index } \\
\hline 0 & 28 & 36 & 41 & $105(52.0 \%)$ \\
\hline 1 to 2 & 21 & 15 & 58 & $94(46.5 \%)$ \\
\hline$\geq 3$ & 0 & 1 & 2 & $3(1.5 \%)$ \\
\hline Total WOMAC score & $56.1 \pm 4.5$ & $57.0 \pm 3.9$ & $56.0 \pm 3.9$ & $56.3 \pm 4.0$ \\
\hline WOMAC pain score & $13.1 \pm 2.1$ & $13.6 \pm 2.1$ & $12.5 \pm 2.5$ & $12.9 \pm 2.3$ \\
\hline WOMAC stiffness score & $3.6 \pm 1.3$ & $3.9 \pm 1.3$ & $3.1 \pm 1.5$ & $3.4 \pm 1.4$ \\
\hline WOMAC function score & $39.3 \pm 3.5$ & $39.4 \pm 3.3$ & $40.4 \pm 3.8$ & $39.9 \pm 3.6$ \\
\hline SF-36 PCS score & $35.0 \pm 4.5$ & $35.1 \pm 5.3$ & $36.0 \pm 4.6$ & $35.5 \pm 4.8$ \\
\hline SF-36 MCS score & $45.6 \pm 5.0$ & $45.5 \pm 6.2$ & $44.4 \pm 3.7$ & $45.0 \pm 4.8$ \\
\hline
\end{tabular}

BMI body mass index, WOMAC Western Ontario and McMaster Universities Osteoarthritis Index, Total WOMAC score WOMAC pain score + WOMAC stiffness score + WOMAC function score, SF-36 36-item short form health survey, PCS Physical Component Summary, MCS Mental Component Summary

\section{Results}

\section{Patient selection and demographic baseline data}

A total of 305 primary TKA patients including 56 simultaneous bilateral, 81 staged bilateral, and 168 unilateral TKA patients were identified during the study period. One hundred three patients were excluded from the study leaving a final of 202 patients recruited for this observational study (Fig. 1). The average age was 69.4 \pm 7.1 years, and average BMI was $26.4 \pm 3.1 \mathrm{~kg} / \mathrm{m}^{2}$. One hundred twenty-eight patients were female (63.4\%).

\section{Clinical outcome measurements}

Overall, $66.8 \%$ of patients (135/202) lost some weight after TKA surgery (Table 2). 20.7\% (42) TKA patients experienced clinically meaningful weight loss (BMI decrease greater than $5 \%$ of preoperative BMI). In the TKA cohort as a whole, mean BMI at 2 years $\left(25.9 \mathrm{~kg} / \mathrm{m}^{2}\right)$ was significantly lower than preoperative BMI $(26.4 \mathrm{~kg} /$ $\mathrm{m}^{2} ; p<0.001$; Table 2). When patients were stratified by surgical strategy, unilateral and simultaneous TKA patients also showed a significantly lower 2-year BMI compared to preoperative BMI $(p<0.001)$. However, staged bilateral TKA patients did not have significantly different preoperative and 2-year BMIs $(p=0.712)$. The patients in each BMI class changed over time (Fig. 2).

The mean 2-year BMI change ( \pm standard deviation) in all TKA patients was $-0.470 \pm 1.067 \mathrm{~kg} / \mathrm{m}^{2}$. Comparison of the BMI percent change between surgical groups showed that BMI change was significantly different between groups (Fig. 3; $p<0.001$ ). A Tukey HSD test revealed simultaneous bilateral TKA patients had a significantly greater percent BMI loss at 2 years compared to the unilateral TKA group $(p=.047)$. Percent BMI loss was significantly lower in the staged bilateral TKA group than in the unilateral TKA group or simultaneous TKA groups ( $p=0.017, p<0.001$ respectively).

BMI and age were found to predict clinically meaningful weight loss and weight gain (Table 3$)$. Increased preoperative BMI was associated with increased odds of clinically meaningful weight loss (odds ratio [OR] 1.27; $p<0.001)$. Increased preoperative BMI was associated with decreased odds of clinically meaningful weight gain (OR $0.70 ; p=0.007$ ). Increased age was associated with increased odds of clinically meaningful weight loss (OR 1.09; $p=0.004$ ) and clinically meaningful weight gain (OR 1.09; $p=0.048$ ). Sex, preoperative WOMAC score, preoperative SF-36 PCS score, and preoperative SF-36 MCS score were not found to be significant predictors of clinically meaningful weight loss or gain.

Clinically meaningful weight loss was associated with lower WOMAC total score $(p<0.001$; Table 4$)$. Similarly, clinically meaningful weight loss was also associated with lower WOMAC pain, function, and stiffness scores $(p=0.001, p=0.002, p=0.015$ respectively). Clinically meaningful weight loss was also associated with higher SF-36 PCS and SF-36 MSC scores $(p<0.001, p=$ 


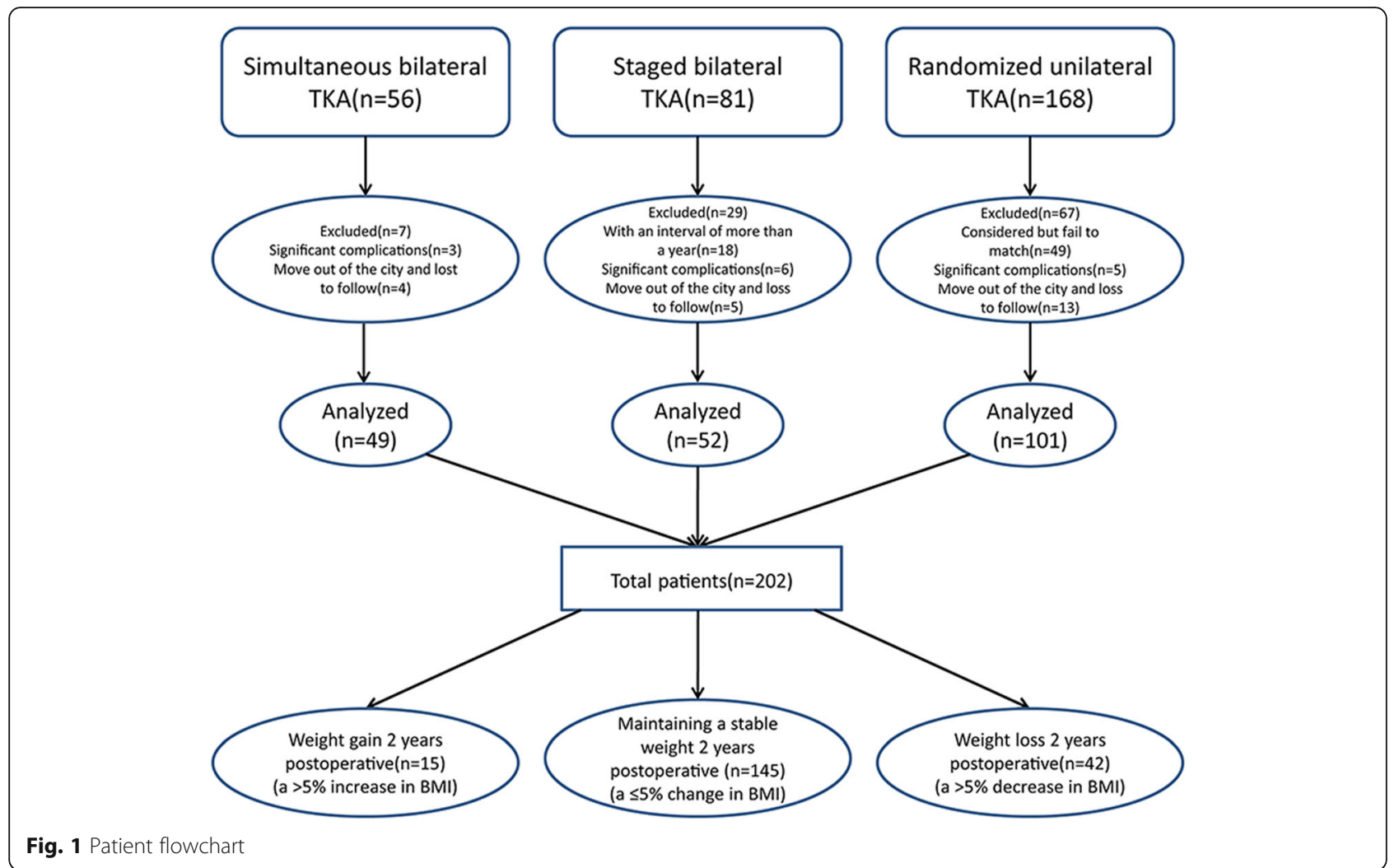

0.025 respectively). Clinically meaningful weight gain was associated with higher WOMAC total score $(p<$ $0.001)$. Clinically meaningful weight gain was associated with higher WOMAC function and stiffness scores $(p<$ $0.001, p<0.001$ respectively). WOMAC pain score was not significantly associated with weight gain. Clinically meaningful weight gain was associated with lower SF-36 PCS and SF-36 MCS scores $(p=0.001, p=0.003$ respectively).

\section{Discussion}

To our knowledge, this is the first study investigating BMI trends in a Chinese cohort after primary TKA. Furthermore, previous studies on BMI trends after TKA have not stratified patients by simultaneous bilateral, staged bilateral, and unilateral TKA. In a Chinese cohort, we found that while most patients lost some weight after TKA surgery, a much smaller subset of patients experienced clinically meaningful weight loss. Unilateral TKA and simultaneous TKA patients significantly lost weight at 2 years after surgery. However, staged bilateral TKA patients did not experience a significant BMI change at 2 years after surgery. Clinically meaningful weight change after TKA was also associated with better patient-reported outcome scores.

We demonstrated that most patients (66.8\%) lose some weight after TKA surgery, and a lower percentage of patients $(20.7 \%)$ lose clinically meaningful weight. However, in our cohort, very few patients $(7.2 \%)$ gained clinically meaningful weight. Our findings are consistent with existing findings. Recently, in a large sample of over 3000 patients, Ast et.al [22] found that $69 \%$ of patients demonstrated no significant change at 2 years after surgery. Dowsey et al. [32] found a clinically meaningful

Table 2 Within-subjects BMI change after TKA surgery

\begin{tabular}{|c|c|c|c|c|}
\hline Parameters & Preoperative (BMI) & 2 years postoperative (BMI) & Change in BMI, mean $(95 \% \mathrm{Cl})$ & $p$ value \\
\hline All TKA patients $(n=202)$ & $26.40 \pm 3.12$ & $25.93 \pm 2.87$ & 0.470 (0.322 to 0.618$)$ & $<0.001^{*}$ \\
\hline Simultaneous bilateral TKA patients $(n=49)$ & $26.15 \pm 2.74$ & $25.20 \pm 2.37$ & 0.950 (0.673 to 1.226$)$ & $<0.001^{*}$ \\
\hline Staged bilateral TKA patients $(n=52)$ & $26.45 \pm 3.43$ & $26.40 \pm 3.19$ & $0.046(-0.201$ to 0.293$)$ & 0.712 \\
\hline Unilateral TKA patients $(n=101)$ & $26.49 \pm 3.14$ & $26.03 \pm 2.87$ & 0.456 (0.237 to 0.676$)$ & $<0.001^{*}$ \\
\hline
\end{tabular}




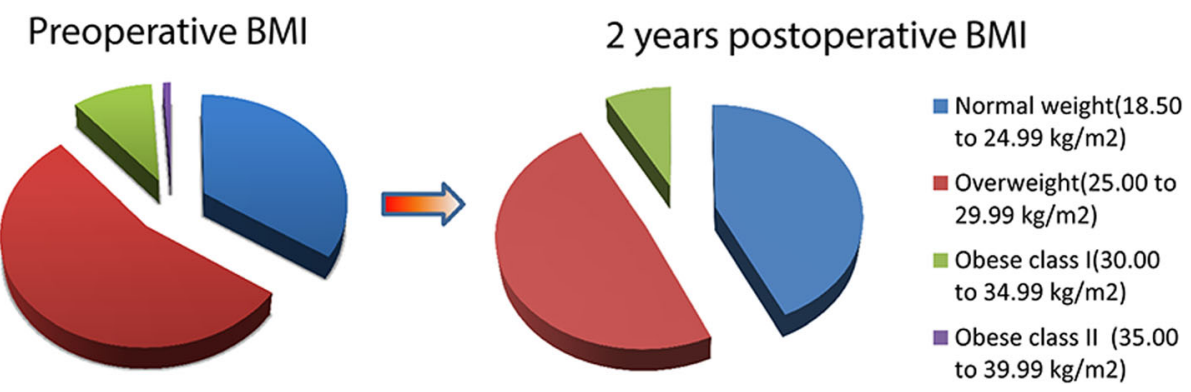

Fig. 2 Patients in each BMI class changed with the follow-up time

weight loss occurred in $12.6 \%$ of patients and $21 \%$ of patients actually experienced weight gain. Kahn et al. [2] compared TKA patients with osteoarthritis patients who did not received TKA surgery; they found no difference in BMI. However, Zeni and Snyder-Mackler [33] compared TKA patients with a healthy control group and found that most patients gained weight 2 years postoperatively. Chang et al. [34] conducted a retrospective review of Korean cohorts and found that $70.2 \%$ of patients maintained a stable BMI and $12.6 \%$ of patients had a clinically meaningful weight loss. A recent systematic review [27] found that existing studies were very low quality and failed to properly address the weight changes following TKA. They were unable to conclude whether weight increases, decreases, or remains the same after TKA.
We also demonstrated that surgery strategy affected BMI change after TKA surgery. Compared with unilateral TKA, simultaneous bilateral TKA patients had significant weight loss. However, staged bilateral TKA surgery was less likely to benefit patients' weight decreasing in a 2-year follow-up. The BMI change in simultaneous bilateral TKA patients was significantly greater than the BMI change in unilateral TKA patients. This may be related to increased mobility following simultaneous bilateral TKA. Unilateral TKA patients may have contralateral osteoarthritis. This osteoarthritis may not be significant to require TKA, but may still limit mobility and function. It is unclear why staged bilateral TKA surgery patients did not show a significant change in BMI at 2 years. The interval period between

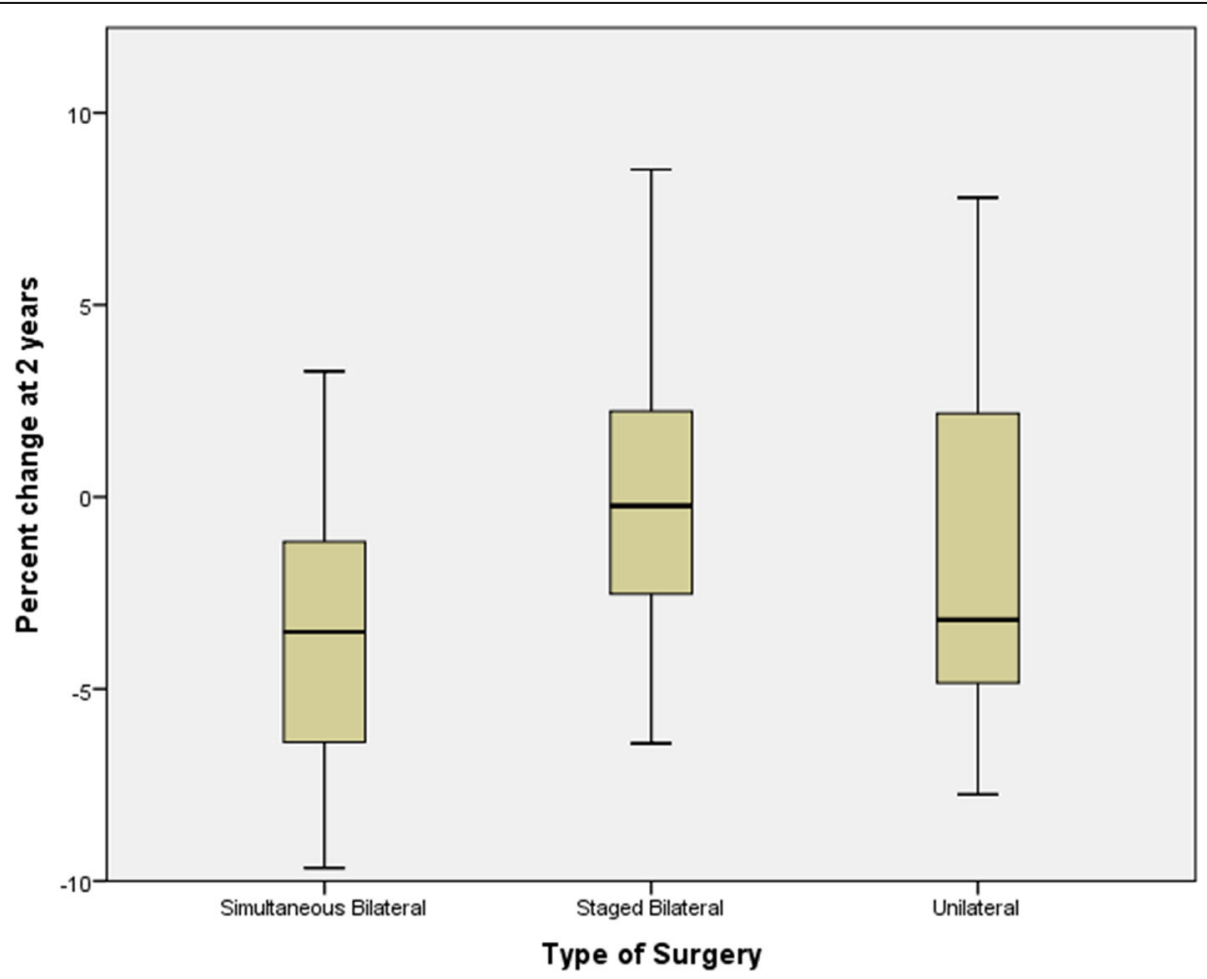

Fig. 3 Box plot showed 2-year postoperative BMI stratified by type of surgery 
Table 3 Logistic regression results evaluating the predictors of clinically meaningful weight loss and gain after TKA surgery

\begin{tabular}{lllll}
\hline Parameters & Weight loss $(n=42)^{ \pm}$ & $p$ value & Weight gain $(n=15)^{£}$ & $p$ value \\
\hline Preoperative BMI & $1.271(1.123$ to 1.440$)$ & $<0.001^{*}$ & $0.698(0.538$ to 0.906$)$ & $0.007^{*}$ \\
Sex & $1.900(0.794$ to 4.548$)$ & 0.149 & $1.161(0.361$ to 3.730$)$ & $1.089(1.001$ to 1.184$)$ \\
Age & $1.088(1.027$ to 1.153$)$ & $0.004^{*}$ & $0.928(0.803$ to 1.073$)$ & $0.048^{*}$ \\
Preoperative total WOMAC score & $1.066(0.968$ to 1.174$)$ & 0.191 & $1.088(0.964$ to 1.230$)$ & 0.313 \\
Preoperative SF-36 PCS score & $0.969(0.893$ to 1.050$)$ & 0.442 & $1.036(0.926$ to 1.159$)$ & 0.173 \\
Preoperative SF-36 MCS score & $1.007(0.927$ to 1.094$)$ & 0.869 & 0.538 \\
\hline
\end{tabular}

${ }^{\mathrm{E}}$ The values are given as the odds ratio, with $95 \%$ confidence interval in parentheses

*Significant difference

operations may contribute to weight trends as patients may be less mobile in between TKA surgeries.

We also wanted to investigate significant predictors that might contribute to BMI change after TKA surgery. We found that preoperative BMI and age predicted clinically meaningful weight loss and weight gain. Increased preoperative BMI was associated with higher likelihood to lose a clinically meaningful weight, and increased age was less likely associated with clinically meaningful weight loss and clinically meaningful weight gain. However, sex, preoperative WOMAC score, preoperative SF-36 PCS score, and preoperative SF-36 MCS score were not found to be significant predictors of clinically meaningful weight loss or gain. Chang et al. [34] found patients with a lower preoperative BMI were associated with more weight gain. Zeni and Snyder-Mackler [33] tried to determine some indicators for weight gain, but they did not find any significant relationships between weight change and educational level, marital status, income level, or activity level prior to surgery. In Ast et al.'s [22] study, they demonstrated that preoperative obesity and female sex were significant predictors of weight loss.

Finally, we examined whether BMI change after surgery was associated with patient-reported outcomes. We found that patients with clinically meaningful weight had significantly better patient-reported outcomes. Patients with clinically meaningful weight gain had inferior outcomes. Our findings are in accordance with some previous studies. Mackie et al. [26] demonstrated that weight gain over $10 \%$ had a negative impact on the SF-36 pain and functional scores. Ast et al. [22] found that weight gain after TKA surgery was associated with inferior scores on the SF-36 PCS score. However, weight loss was not associated with better outcome scores despite seeing better outcome scores in total hip arthroplasty patients with weight loss. Additionally, many studies have demonstrated that weight gain after TKA was associated with higher incidence of complications and shorter time to revision [30, 35, 36]. Given the evidence presented above, orthopedic surgeons should counsel patients to avoid weight gain after TKA to reduce the adverse influences of weight gain on the clinical outcomes.

Our findings need to be interpreted in light of some potential limitations. Firstly, our sample size is small compared to some previous studies. However, we were most interested in the bilateral TKAs, limiting our sample size as this is not common practice at our hospital. Secondly, patient-reported WOMAC and SF-36 scores are subjective surveys which do suffer from bias. Furthermore, some patients may misinterpret questions on surveys; however, an independent nurse provided interpretation and instruction on the surveys for all the patients. Thirdly, our cohorts were comprised completely of Chinese individuals, and the mean BMI was notably lower than has been reported in many Western cohorts. While this is one unique aspect of our cohort, our findings

Table 4 Multiple linear regression results evaluating the effects of clinically meaningful weight loss and gain on the outcomes after TKA surgery

\begin{tabular}{lllll}
\hline Parameters & Weight loss $(n=42)^{*}$ & $p$ value & Weight gain $(n=15)^{*}$ & $p$ value \\
\hline WOMAC total score & $11.50 \pm 2.85$ & $<0.001^{*}$ & $17.47 \pm 1.81$ & $<0.001^{*}$ \\
WOMAC pain score & $1.40 \pm 0.63$ & $0.001^{*}$ & $2.07 \pm 0.80$ & 0.120 \\
WOMAC function score & $9.45 \pm 2.60$ & $0.002^{*}$ & $13.53 \pm 2.17$ & $<0.001^{*}$ \\
WOMAC stiffness score & $0.64 \pm 0.66$ & $0.015^{*}$ & $1.87 \pm 0.92$ & $<0.001^{*}$ \\
SF-36 PCS score & $49.38 \pm 2.02$ & $<0.001^{*}$ & $42.4 \pm 3.14$ & $0.001^{*}$ \\
SF-36 MCS score & $50.83 \pm 8.19$ & $0.025^{*}$ & $43.80 \pm 3.19$ & $0.003^{*}$ \\
\hline
\end{tabular}

${ }^{¥}$ Values are given as mean and standard deviation

*Significant difference 
may not be generalizable to groups of different racial proportions, cultures, diet habits, and esthetic standards. Though these potential limitations exist, our present study provides some evidence regarding the BMI changes, predictors, and outcomes in simultaneous bilateral, staged bilateral, and unilateral TKA Chinese patients.

\section{Conclusion}

In a Chinese cohort, most patients lost weight after TKA surgery; however, fewer patients lost clinically meaningful weight. Simultaneous bilateral and unilateral TKA patients had significantly decreased BMI at 2 years. Clinically meaningful weight loss after TKA surgery was associated with more satisfied patient-reported outcomes. TKA patients should be advised regarding that weight loss after TKA can improve outcomes.

\section{Abbreviations}

BMI: Body mass index; MCS: Mental component summary; PCS: Physical component summary; SF-36: 36-item short form health survey; TKA: Total knee arthroplasty; WOMAC: Western Ontario and McMaster Universities Osteoarthritis Index

\section{Acknowledgements}

We really appreciate all the nursing staff of our orthopedic department for helping collect and record all data.

\section{Funding}

This work was supported by the National Natural Science Foundation of China (NSFC), grant number (81572632).

\section{Availability of data and materials}

The dataset supporting the conclusions of this article is available on request-please contact the corresponding author Dr. Weixu Li and Dr. Guodong Li. Administrative permission was received from Shanghai Tenth People's Hospital Affiliated to Tongji University to access the medical records.

\section{Authors' contributions}

$P Z, W L$, and GL designed the study and drafted the manuscript. PZ, JY, and $\mathrm{KL}$ accumulated the data. $\mathrm{DY}, \mathrm{KL}$, and $\mathrm{PZ}$ analyzed the data. $\mathrm{WL}$ and $J Y$ proof the English language. All authors read and approved the final manuscript.

\section{Ethics approval and consent to participate}

The Ethics Committee of Shanghai Tenth People's Hospital Affiliated to Tongji University approved the study protocol. Written informed consent was obtained from all patients enrolled in the investigation.

\section{Consent for publication}

Not applicable.

\section{Competing interests}

The authors declare that they have no competing interests.

\section{Publisher's Note}

Springer Nature remains neutral with regard to jurisdictional claims in published maps and institutional affiliations.

\section{Author details}

${ }^{1}$ Department of Orthopedic Surgery, Second Affiliated Hospital of Zhejiang University, School of Medicine, 88 Jiefang Road, Hangzhou 310009, People's Republic of China. 'Department of Orthopedic Surgery, The Tenth People's Hospital Affiliated to Tongji University, 301 Yanchang Rd, Shanghai 200072, People's Republic of China. ${ }^{3}$ Department of Orthopedic Surgery, University of Texas Southwestern Medical Center, Dallas, TX, USA.
Received: 30 September 2018 Accepted: 3 May 2019

Published online: 02 July 2019

\section{References}

1. Pulido L, Parvizi J, Macgibeny M, Sharkey PF, Purtill JJ, Rothman RH, Hozack WJ. In hospital complications after total joint arthroplasty. J Arthroplast. 2008:23-6:139-45.

2. Kahn TL, Snir N, Schwarzkopf R. Does body mass index decrease over time among patients who undergo total knee arthroplasty compared to patients with osteoarthritis? Data from the osteoarthritis initiative. J Arthroplast. 2016; 31(5):971-5.

3. Losina E, Walensky RP, Kessler CL, Emrani PS, Reichmann WM, Wright EA, Holt HL, Solomon DH, Yelin E, Paltiel AD. Cost-effectiveness of total knee arthroplasty in the United States: patient risk and hospital volume. Arch Intern Med. 2009;169(12):1113.

4. Ethgen O, Bruyere O, Richy F, Dardennes C, Reginster J-Y. Health-related quality of life in total hip and total knee arthroplasty. J Bone Joint Surg Am. 2004;86(5):963-74

5. Alvi HM, Mednick RE, Krishnan V, Kwasny MJ, Beal MD, Manning DW. The effect of BMI on 30 day outcomes following total joint arthroplasty. J Arthroplast. 2015;30(7):1113-7.

6. Kremers HM, Visscher SL, Kremers WK, Naessens JM, Lewallen DG. Obesity increases length of stay and direct medical costs in total hip arthroplasty. Clin Orthop Relat Res. 2014;472(4):1232-9.

7. Kremers HM, Visscher SL, Kremers WK, Naessens JM, Lewallen DG. The effect of obesity on direct medical costs in total knee arthroplasty. Bone Joint J. 2014;96(9):718-24

8. Bowditch M, Villar R. Do obese patients bleed more? A prospective study of blood loss at total hip replacement. Ann R Coll Surg Engl. 1999:81-3:198.

9. Naziri Q, Issa K, Malkani AL, Bonutti PM, Harwin SF, Mont MA. Bariatric orthopaedics: total knee arthroplasty in super-obese patients (BMl>50 kg/m2). Survivorship and complications. Clin Orthop Relat Res. 2013;471(11):3523-30.

10. Wagner ER, Kamath AF, Fruth K, Harmsen WS, Berry DJ. Effect of body mass index on reoperation and complications after total knee arthroplasty. J Bone Joint Surg Am. 2016;98(24):2052-60.

11. Crawford DA, Berend KR, Nam D, Barrack RL, Adams JB, Lombardi AV. Low rates of aseptic tibial loosening in obese patients with use of high viscosity cement and standard tibial tray: 2-year minimum follow-up. J Arthroplast. 2017;32(9S):S183-6.

12. Bonnefoy-Mazure A, Martz P, Armand S, Sagawa Y, Suva D, Turcot K Miozzari HH, Lübbeke A. Influence of body mass index on sagittal knee range of motion and gait speed recovery 1-year after total knee arthroplasty. J Arthroplast. 2017;32(8):2404-10.

13. Issa K, Pivec R, Kapadia BH, Shah T, Harwin SF, Delanois RE, Mont MA. Does obesity affect the outcomes of primary total knee arthroplasty? J Knee Surg. 2013;26(02):089-94.

14. Malinzak RA, Ritter MA, Berend ME, Meding JB, Olberding EM, Davis KE. Morbidly obese, diabetic, younger, and unilateral joint arthroplasty patients have elevated total joint arthroplasty infection rates. J Arthroplast. 2009;24-6:84-8.

15. OToole P, Maltenfort MG, Chen AF, Parvizi J. Projected increase in periprosthetic joint infections secondary to rise in diabetes and obesity. J Arthroplast. 2016;31(1):7-10.

16. Mantilla CB, Horlocker TT, Schroeder DR, Berry DJ, Brown DL. Risk factors for clinically relevant pulmonary embolism and deep venous thrombosis in patients undergoing primary hip or knee arthroplasty. Anesthesiology. 2003; 99(3):552-60.

17. Zhang Z-h, Shen B, Yang J, Z-k Z, F-x P. Risk factors for venous thromboembolism of total hip arthroplasty and total knee arthroplasty: a systematic review of evidences in ten years. BMC Musculoskelet Disord. 2015;16(1):24.

18. Namba RS, Paxton L, Fithian DC, Stone ML. Obesity and perioperative morbidity in total hip and total knee arthroplasty patients. J Arthroplast. 2005:20:46-50.

19. Springer BD, Parvizi J, Austin M, Backe H, Della Valle C, Kolessar DJ, Kreuzer S, Malinzak R, Masri B, McGrory BJ. Obesity and total joint arthroplasty a literature based review. J Arthroplast. 2013;28(5):714-21.

20. Donovan J, Dingwall I, McChesney S. Weight change 1 year following total knee or hip arthroplasty. ANZ J Surg. 2006;76(4):222-5.

21. Heisel C, Silva M, Rosa MAD, Schmalzried TP. The effects of lowerextremity total joint replacement for arthritis on obesity. Orthopedics. 2005;28(2):157-9. 
22. Ast MP, Abdel MP, Y-y L, Lyman S, Ruel AV, Westrich GH. Weight changes after total hip or knee arthroplasty. J Bone Joint Surg Am. 2015;97(11):911-9.

23. Kandil A, Novicoff WM, Browne JA. Obesity and total joint arthroplasty: do patients lose weight following surgery? Phys Sportsmed. 2013;41(2):34-7.

24. Stets K, Koehler SM, Bronson W, Chen M, Yang K, Bronson MJ. Weight and body mass index change after total joint arthroplasty. Orthopedics. 2010:336:386.

25. Teichtahl AJ, Quirk E, Harding P, Holland AE, Delany C, Hinman RS, Wluka AE, Liew SM, Cicuttini FM. Weight change following knee and hip joint arthroplasty-a six-month prospective study of adults with osteoarthritis. BMC Musculoskelet Disord. 2015;16(1):137.

26. Mackie A, Muthumayandi K, Shirley M, Deehan D, Gerrand C. Association between body mass index change and outcome in the first year after total knee arthroplasty. J Arthroplast. 2015;30(2):206-9.

27. Inacio MC, Kritz-Silverstein D, Paxton EW, Fithian DC. Do patients lose weight after joint arthroplasty surgery? A systematic review. Clin Orthop Relat Res. 2013:471(1):291-8.

28. McConnell S, Kolopack P, Davis AM. The Western Ontario and McMaster universities osteoarthritis index (WOMAC): a review of its utility and measurement properties. Arthritis Care Res (Hoboken). 2001;45(5):453-61.

29. Lins L, Carvalho FM. SF-36 total score as a single measure of health-related quality of life: scoping review. SAGE open medicine 2016;4: 2050312116671725.

30. Organization WH. Obesity: preventing and managing the global epidemic World Health Organization, 2000.

31. Food, Administration D. Guidance for industry developing products for weight management. Washington, DC: Food and Drug Administration 2007.

32. Dowsey M, Liew D, Stoney J, Choong P. The impact of pre-operative obesity on weight change and outcome in total knee replacement. Bone Joint J. 2010;92-4:513-20.

33. Zeni J, Snyder-Mackler L. Most patients gain weight in the 2 years after total knee arthroplasty: comparison to a healthy control group. Osteoarthr Cartil. 2010;18(4):510-4.

34. Chang MJ, Kang YG, Chang CB, Seong SC, Kim TK. The patterns of limb length, height, weight and body mass index changes after total knee arthroplasty. J Arthroplast. 2013;28(10):1856-61.

35. Lim CT, Goodman SB, Huddleston Jl, Harris AH, Bhowmick S, Maloney WJ, Amanatullah DF. Weight gain after primary total knee arthroplasty is associated with accelerated time to revision for aseptic loosening. J Arthroplast. 2017;32(7):2167-70.

36. Electricwala AJ, Jethanandani RG, Narkbunnam R, Huddleston Jl, Maloney WJ, Goodman SB, Amanatullah DF. Elevated body mass index is associated with early total knee revision for infection. J Arthroplast. 2017;32(1):252-5.

Ready to submit your research? Choose BMC and benefit from:

- fast, convenient online submission

- thorough peer review by experienced researchers in your field

- rapid publication on acceptance

- support for research data, including large and complex data types

- gold Open Access which fosters wider collaboration and increased citations

- maximum visibility for your research: over $100 \mathrm{M}$ website views per year

At BMC, research is always in progress.

Learn more biomedcentral.com/submissions 